\title{
Factores que inciden la percepción de la imagen de un político en el escenario electoral
}

\section{Factors that affect the perception of the image of a politician in the electoral stage}

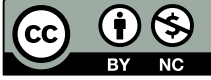

\author{
Ec. Jorge Guido Sotomayor Pereira, M.B.A. ${ }^{1}$ \\ jsotomayor@utmachala.edu.ec \\ Egda. Magaly Anabell Barrezueta Rodriguez \\ mabarrezueta_est@utmachala.edu.ec \\ Ing. Luis Felipe Brito Gaona, $\mathrm{Mgs}^{1}$ \\ Ibrito@utmachala.edu.ec \\ Ing. Bill Jonathan Serrano Orellana, M.A.E. \\ bjserrano@utmachala.edu.ec
}

Recibido: 1/07/2017, Aceptado: 1/09/2017

\begin{abstract}
RESUMEN
En los últimos tiempos los políticos no solo buscan presentar sus propuestas de trabajo, también es su prioridad cuidar la imagen que proyectan a sus votantes, permitiéndoles promover su popularidad en el mercado electoral; la imagen no se vincula con la estética más bien es el poder que ejerce su presencia ante los demás; la mayoría de las veces éstas estrategias son utilizadas en las campañas de obtención de votos, favoreciendo el contacto directo con el elector siendo este el mayor desafío en la contienda electoral que catapulta al personaje en la palestra política. La imagen se crea de acuerdo al medio en el que se desarrolla, el rol que se asume debe simbolizar la realidad que viven los electores, si se omite esta particularidad la imagen no va a tener sustento suficiente frente a los votantes que se desean captar. Los políticos planifican acciones que ayudan a afianzar su imagen utilizando estrategias de marketing, las cuales permiten dinamizar las formas de comunicación y promoción; partiendo desde la planificación hacia la evaluación de los procesos y control de resultados. El presente trabajo investigativo tiene por objetivo determinar los factores que influyen en la percepción de la imagen de un político en el escenario electoral; se considera que la imagen es el sello personal de un político y se debe cimentar en su propia esencia, los símbolos, gestos, posturas, comportamiento y acciones repercute en la aceptación de su mercado; para afianzar la imagen política se deberá crear una buena opinión pública basada en la credibilidad e integridad que ayude a diferenciarse de sus homólogos, utilizando recursos simbólicos que ayuden a la consolidación de la imagen del político y su ideología.
\end{abstract}

Palabras clave: Marketing Político, Imagen Política, Comunicación Política, Percepción

\footnotetext{
${ }^{1}$ Profesor titular Universidad Técnica de Machala. Ecuador
} 
Revista Ciencia \& Tecnología No. 16, 31 de octubre de 2017 ISSN impreso: 1390 - 6321

\section{Jornada de Investigación Universidad Tecnológica Empresarial de Guayaquil}

\begin{abstract}
In recent times, politicians not only seek to present their work proposals, it is also their priority to take care of the image they project to their voters, allowing them to promote their popularity in the electoral market; the image is not linked to aesthetics, rather it is the power exercised by its presence before others; Most of the time these strategies are used in campaigns to obtain votes, favoring direct contact with the voter being this the biggest challenge in the electoral contest that catapults the character in the political arena. The image is created according to the environment in which it is developed, the role that is assumed must symbolize the reality that the voters live, if this particularity is omitted, the image will not have enough support in front of the voters that are to be captured. The politicians plan actions that help to strengthen their image using marketing strategies, which allow to stimulate the forms of communication and promotion; starting from the planning to the evaluation of the processes and control of results. The objective of this research work is to determine the factors that influence the perception of the image of a politician in the electoral scenario; it is considered that the image is the personal stamp of a politician and should be based on its own essence, the symbols, gestures, postures, behavior and actions have an impact on the acceptance of its market; To strengthen the political image, a good public opinion based on credibility and integrity must be created to help differentiate from its counterparts, using symbolic resources that help consolidate the image of the politician and his ideology.
\end{abstract}

Keywords: Political Marketing, Political Image, Political Communication, Perception

\title{
Introducción
}

La imagen que proyecta un político es el primer mensaje que comunica a su pueblo. La conjugación de la imagen que desea transmitir y la imagen que lo sustenta permitirá mejorar el dialogo político entre las partes interesadas y así garantizar el éxito en el escenario electoral (Orejuela, 2009). La construcción de la imagen permite al personaje político posicionarse en la mente de sus votantes y romper figuras estereotipadas que han trascendido a las contiendas electorales.

Desde el punto de vista pragmático-electoral las características más importantes que deben cultivar los políticos son la credibilidad y la percepción que tenga el electorado sobre él, ésta permite a los votantes captar la imagen que transfieren, facilitando la comunicación (Sanmartin, 2007). La construcción de la imagen involucra aspectos personales que un político debe fortalecer como dominar su lenguaje oral, visual y simbólico; son lógicas básicas de persuasión inconsciente que debe manejar para promover el interés de su público (Richard, 2008). La buena imagen de un político ampliamente difundida dentro de las campañas de comunicación mejora el nivel de aceptación y asegura su permanente favoritismo, considerando que el electorado se identifica con la imagen que presenta el candidato como su forma de ser, de vestirse y su estilo de vida (Merino, 2015). Se debe construir una imagen sólida y en relación con las tendencias ideológicas de sus electores, los asesores políticos y expertos en marketing deben adecuar tácticas basadas en esta pluralidad.

Por tal motivo, para el desarrollo de la imagen política se debe implementar herramientas relacionadas con el Marketing tradicional, la aplicación de estas técnicas dentro de su programa político ha permitido que los candidatos y sus partidos desarrollen estrategias para captar la atención del pueblo y la intención del voto de 


\section{Jornada de Investigación Universidad Tecnológica Empresarial de Guayaquil}

su electorado para ganar en las elecciones populares.

Hoy por hoy los instrumentos de marketing han sido utilizados en el medio político, muchas campañas aseguran su éxito utilizando tácticas de marketing que permiten a las organizaciones políticas persuadir al mayor número de militantes, simpatizantes o adherentes los cuales garantizan el respaldo necesario para partidos políticos y sus candidatos (Salas \& Serratore, 2015).

Estableciendo estrategias de marketing mix y publicidad dentro de la política, permite construir la imagen, credibilidad e identidad en una mezcla de propaganda y relaciones públicas que favorezca la aceptación del personaje político (Bustamante, Blanc, \& Espinoza, 2015).

Bajo este contexto, en los últimos años el escenario político ha evolucionado con la aparición de figuras en las contiendas electorales. En la actualidad las personas que aspiran a cargos de elección popular, recurren a las herramientas del marketing político para dar solución a su principal problema: Como llegar al cliente (electorado) (Barrientos, 2010).

El propósito del presente estudio es conocer los factores que inciden la percepción de la imagen de un político en el escenario electoral, este trabajo se constituye en una investigación que permitirá establecer conclusiones relativas al tema y conocer los elementos que contribuyen la construcción de la imagen política, esta estrategia es utilizada por personas y partidos políticos que desean alcanzar un cargo público de elección popular.

\section{Definiciones de Marketing Comercial y Marketing Político.}

La constante evolución del mercado electoral y la inserción de profesionales de marketing en la política, ha permitido el perfeccionamiento de las formas de comunicación tradicional hacia las pericias modernas, encaminando los esfuerzos para alcanzar los objetivos establecidos y utilizando recursos de forma eficiente acordes a las nuevas tecnologías; de esta manera los políticos se están familiarizando con la inclusión de nuevos colaboradores dentro de su grupo de trabajo tales como asesores de imagen, sociólogos, psicólogos y mercadólogos que se involucran directamente en el terreno político; puesto que el ciudadano no vota por el candidato sino que lo compra psicológicamente (Merino, 2015).

Bajo esta premisa autores clásicos como Stanton, Etzel, \& Walker (2007) instauran un concepto de marketing puntualizando que: Es un sistema total de actividades de negocios ideado para planear productos satisfactores de necesidades, asignarles precio, promoverlos y distribuirlos a los mercados meta, a fin de lograr los objetivos de la organización. Esta definición tiene dos implicaciones significativas: Enfoque y Duración.

En el libro Fundamentos de Marketing expertos en mercadotecnia como (Kotler \& Armstrong, 2012) definen a esta disciplina como el proceso mediante el cual las compañías crean valor para sus clientes y establecen relaciones sólidas con ellos para obtener a cambio valor de éstas. Con esta inferencia los autores especifican el objetivo del marketing dentro del ámbito en el que se desarrolla. 


\section{Jornada de Investigación Universidad Tecnológica Empresarial de Guayaquil}

Con estas perspectivas enfocadas en la mercadotécnica comercial, se considera que entre el Marketing y la Política ha existido un vínculo desde la antigüedad, en 1953 Maquiavelo considerado pionero en la concepción de la ciencia política, desarrolló una sofisticada teoría sobre el papel de la persuasión política como alternativa al uso de la fuerza, en su obra clásica El Príncipe (Benítez \& Salas, 2016). De esta manera se destaca la importancia del marketing dentro de política, la conjugación de estas ciencias forma los cimientos de lo que ahora conocemos como "marketing político".

Los autores (Lerma, Bárcena, \& Vite, 2011) expresan que el marketing político comprende el conjunto de técnicas que permiten primero, captar y conocer las necesidades, deseos y aspiraciones de una sociedad (mercado electoral), y después analizar y elaborar el diagnostico correspondiente a fin de establecer, con base en esas necesidades un programa ideológico y de acción (propuesta) que pretenda solucionarlas, ofreciéndole al mercado incentivos (con candidatos, plataformas, planes, etc.) que materialicen dicho programa al que se apoya e impulsa por medio de la publicidad política (propaganda) y acciones de proselitismo.

El autor (Barranco, 2012) define al Marketing Político como el conjunto de técnicas que permiten captar las necesidades que un mercado electoral tiene, estableciendo, en base a esas necesidades, un programa ideológico que las satisfaga y ofreciéndole un candidato que personalice dicho programa y al que se apoya e impulsa a través de la publicidad.

La palestra política posee una base fortalecida en el marketing con la implementación de sus tácticas, pudiendo lograr que se impulse nuevas leyes de gobierno, ganar adeptos para un determinado partido político o conseguir el mayor número votantes que respalden a sus candidatos a alcanzar puestos de elección popular dentro de las administraciones públicas (Castro, 2012). El marketing político se entiende como un instrumento al servicio de los partidos como de sus electores, al emplear técnicas para promocionar la imagen, ideología o los mismos candidatos (Sánchez, 2013).

\section{Surgimiento del Marketing Político}

Algunos líderes consideran la posibilidad que el Marketing Político podría ayudarlos a vencer las elecciones, lo que ellos sí aseguran es que esta ciencia les da la inmortalidad logrando quedarse en el alma de sus electores. John F. Kennedy icono de la política en América lo especificaba como el arte de adentrarse en la mente de los hombres de manera que puedan darse cuenta del esplendor de nuestros ideales (Alonso Coto \& Adell, 2011).

El marketing político nace en Estados Unidos a inicios de la década de los cincuenta, a este país se lo considera como el pionero en la concepción de la mercadotecnia política debido al predominio de los casos políticos más emblemáticos en la historia (Castro, 2012). Según (Sánchez, 2013) Innumerables políticos a lo largo de la historia destacaron el uso de las estrategias de marketing para alcanzar sus metas, políticos estadounidenses como Jackson en 1828, Roosevelt en 1936 y Truman en 1948 son los precursores en la implementación de técnicas de marketing para el desarrollo de sus campañas (Salas L. \& Serratore, 2015).

El surgimiento del Marketing Político y la aplicación de sus estrategias lo originó el general Dwight Eisenhower en la campaña que lo llevo a la Casa Blanca en 1952, 140 


\section{Jornada de Investigación Universidad Tecnológica Empresarial de Guayaquil}

siendo este el primer candidato republicano que contrató los servicios de la agencia de publicidad Ilamada BBDO, John F. Kennedy y Richard Nixon protagonizaron el primer debate televisivo en 1960 marcando un antes y un después en la historia de la política en América, recalcando la influencia de la imagen como parte de su mensaje político, Ronald Reagan fortaleció su gran carisma y atractivo personal en 1980 para diferenciarse de sus competidores mostrando un aspecto más presidencial; en el ámbito internacional Françoise Mitterrand (Francia) y Felipe González (España) utilizaron la video-política y video-mediatización masivas para llevar su imagen política a su público objetivo; en 1988 George Bush y Michael Dukakis fueron asistidos por asesores de imagen para reforzar sus perfiles, en 1990 Bill Clinton político americano junto a homólogos internacionales como Tony Blair de Gran Bretaña y el español José María Aznar afianzaron su imagen popular con la participación en programas de televisión para llegar a su audiencia; en el año 2000 la mayoría de políticos comenzaron a utilizar las técnicas de Marketing Político como: campañas de imagen, grupos focales, spots publicitarios y sondeos de opinión. En el 2007 Barack Obama fue catalogado como el primer presidente 2.0 basando su campaña en el internet, redes sociales y comunicación telefónica; este candidato dio un giro considerable en las formas de comunicación utilizando su imagen (Merino, 2015).

Se concibe que el marketing político es la adaptación de las herramientas del mundo empresarial hacia la política, aquí también se utilizan vocablos claves como producto, servicio, mercado o marca; considerando que el marketing político es aproximar un producto, una ideología o un servicio a los electores puestos que ellos compran los beneficios que pueden obtener de sus políticos.

Se considera al marketing político como el vínculo que permite alcanzar objetivos establecidos, con su implementación promueve y facilita la relación entre los políticos y su público (Bustamante, Blanc, \& Espinoza, 2015).

\section{Objetivo del Marketing Político}

Para alcanzar los objetivos planteados, se establecen actividades relacionadas al Marketing Político entre los candidatos y sus partidos, con la finalidad de obtener la mayor cantidad de votos posibles; utilizando el Marketing Interno para la selección de voluntarios y candidatos, el comportamiento del consumidor permite conocer a los votantes potenciales, el análisis del producto político y las relaciones públicas son herramientas que permitirán alcanzar las metas primigenias, las cuales son ganar las elecciones (Alonso Coto \& Adell, 2011). Los actores son aquellas personas consideradas dentro de los objetivos; en el Marketing Comercial intervienen los oferentes, la mercancía y los demandantes mientras que en el Marketing Político se involucran los gobernantes, el poder y los gobernados (Lerma, Bárcena, \& Vite, 2011).

El objetivo del marketing político, es lograr vender una ideología, un programa de trabajo y un candidato; desarrollando actividades que permitan influir en la opinión de los electores, para posicionar al partido y al candidato político con respecto a sus competidores (Bustamante, Blanc, \& Espinoza, 2015).

Bajo este contexto, con la implementación de las herramientas de marketing político se puede plasmar los objetivos propuestos como construir y definir la imagen pública, estudiar el mercado electoral, captar votos, reforzar el mensaje publicitario, mejorar la comunicación, fortalecer la propaganda política y articular un discurso acorde al 
Revista Ciencia \& Tecnología No. 16, 31 de octubre de 2017 ISSN impreso: 1390 - 6321

\section{Jornada de Investigación Universidad Tecnológica Empresarial de Guayaquil}

mercado al que se dirigen los esfuerzos.

\section{P del Marketing Político}

De acuerdo a lo expuesto por Bustamante, Blanc, \& Espinoza (2015), el marketing político emplea los principios que aplica el marketing moderno, basado en el mix de las (seis P), "el producto" lo representa el candidato, "el precio" sería la ganancia o beneficio que recibe el votante por ejercer su derecho a sufragar por el candidato 0 partido político preferido, "la plaza o distribución" son los diferentes canales utilizados para dar a conocer al candidato y a su partido político, "la promoción" se refiere a la comunicación integral que recibe el electorado para crear la mejor imagen y credibilidad posible del candidato y el partido político, "las personas" están representadas por el equipo de trabajo que colabora con las aplicación de las estrategias establecidas y "los procesos" tiene que ver con la logística y la ejecución de las diversas actividades que se emprendan, antes durante y después de la campaña (pág. $s / n$ ).

El marketing político sustenta su proceder bajo el principio de "orientar al votante" para generar influencia sobre la opinión pública y favorecer a sus intereses; dentro de las estrategias de comunicación incorporan al marketing electoral con la finalidad de presentar la imagen perfecta y convertir a sus políticos en personajes mediáticos que representen las necesidades de sus votantes (García \& Jaimes, 2010).

Actualmente muchos políticos han consolidado su éxito implementando campañas basadas en estrategias y tácticas de mercadotecnia, recurriendo también al uso del internet y la comunicación directa con el elector lo cual les facilita mostrar una imagen innovadora y fresca que ayude a potencializar sus cualidades y atributos hacia su público objetivo (Salas \& Serratore, 2015). Dentro del desarrollo del marketing político se realizan procesos que permiten destacar sus ventajas competitivas en el mercado electoral, generar presencia y promover su imagen política.

\section{Definición de imagen}

De acuerdo a la (Real Academia Española, 2014) imagen proviene del latín Imago Inis y la define como: "Figura, representación, semejanza y apariencia de algo". La imagen Pública es el "Conjunto de rasgos que caracterizan ante la sociedad a una persona o entidad".

Según Capriotti (2013) en su libro Planificación Estratégica de la Imagen Corporativa; señala que, en la actualidad, la palabra imagen se utiliza para definir gran cantidad de cosas o fenómenos. Debido a la polisemia del término, se puede comprobar en las diversas definiciones.

Teniendo en cuenta lo expresado por Capriotti (2013): La profusión de significados influye que su utilización sea confusa dentro del ámbito de la comunicación, puede haber discrepancia entre imagen-símbolo, imagen global, imagen de las actividades, imagen de los productos, imagen de los hombres e imagen como apariencia del hecho. Además, se diferencia entre la autoimagen y la imagen social; estableciendo cinco tipos los cuales son: imagen del espejo, imagen corriente, imagen deseada, imagen corporativa e imagen múltiple. También se enfatiza la imagen real, potencial y óptima.

Costa (2003) recopila expresiones y define: imagen gráfica, imagen visual, imagen 142 


\section{Jornada de Investigación Universidad Tecnológica Empresarial de Guayaquil}

material, imagen mental, imagen de empresa, imagen de marca, imagen corporativa e imagen global. Otros autores mencionan a la imagen depositada, la imagen deseada y la imagen difundida y concretan tres extensiones: la autoimagen, la imagen intencional y la imagen pública. (Villafañe, 2002).

A finales de los años ochenta surgió la convergencia entre múltiples disciplinas para estudiar la imagen partiendo desde la filosofía, las ciencias políticas, la psicología y los medios de comunicación. En la actualidad se implementa la neurociencia dentro de la política, la cual consiste en localizar zonas cerebrales que se activan con la percepción de la imagen, la misma surge como una forma de comunicación aprehensiva y que sirve para captar objetos (Mc Phail Fanger, 2014).

\section{Construcción y Percepción de la Imagen Política}

Denominamos percepción a la forma en que captamos la realidad que nos rodea encerrando las experiencias sensoriales de las personas. Existe cierta distinción entre la percepción la cual se vincula a los objetos, mientras que la sensación es una experiencia subjetiva asociada a los sentidos. Los individuos pueden tener diversas formas de percepción frente a un estímulo, por tal motivo los mercadólogos se esmeran por comunicar sus mensajes de forma constante y que conecte directamente con el receptor (Ponce, Besanilla, \& Rodríguez, 2012).

Cuando hablamos de "imagen" en marketing político engloba su discurso, personalidad y sobre todo su ideología, es decir, toda la campaña política. La imagen que proyecte un candidato deberá ser creíble y fomentar confianza para genera respeto de sus contendientes electorales y convencer a su público elector con sus propuestas de gobierno. Todo esto debe se debe abarcar dentro de la comunicación política del candidato (Richard, 2008). La imagen que proyecta un político, un partido o un gobierno; contribuye al posicionamiento en la mente de su elector. La constante comunicación en diferentes medios de información permitirá a los ciudadanos sentirse representados y beneficia a los líderes quienes aseguran su contante vigencia dentro del escenario político.

La imagen simboliza la estrategia principal de comunicación, la misma se utiliza en todas las fases de las campañas resaltando la vigencia política, personalidad, profesión, atributos, carisma, compromiso y fortalezas que debería mostrar un político a su público. Formular palabras de clemencia construye la imagen como un proceso subjetivo que conecta con la empatía de su público; el uso de afiches, volantes y trípticos se convierten en el eje central de las campañas los cuales permiten especificar partes de la imagen que debería destacar un político y establecer mecanismos de comunicación global para captar a sus votantes potenciales. La personalización se fundamente en la imagen, la cual debe impactar a la opinión pública; el argumento político y las cualidades del candidato constituyen la imagen personal la cual se convierte en el símbolo y el mensaje dentro de la comunicación electoral (Orejuela, 2009).

Para la correcta construcción de la imagen pública se debe estudiar al mercado al que va dirigido el programa político, hay que tomar en cuenta el entorno, la región, la población, el tipo de vestimenta, tradiciones, estilo de vida, factores personales, edad, cultura y la religión para asegurar la aceptación inmediata con la imagen que proyecta; es imperativo plasmar una imagen política alejado de la superficialidad, caprichos, vanidad y frivolidad (Ponce, Besanilla, \& Rodríguez, 2012). 


\section{Jornada de Investigación Universidad Tecnológica Empresarial de Guayaquil}

La imagen que proyecta un político es muy importante dentro de la relación que se establece con su público esta debe estar relacionada con sus antecedentes personales, su preparación, su ideología, su léxico, la etiqueta y protocolo, su masculinidad o femineidad sin confundir con los estereotipos de belleza física (Bustamante, Blanc, \& Espinoza, 2015). Cabe mencionar que los electores prefieren políticos homologados con el común de su clase social.

La imagen desborda límites de lo físico a lo abstracto partiendo de las percepciones, sensaciones, estímulos y conductas es decir la subjetividad humana. La imagen va desde lo tangible hasta llegar a la memoria, desde la representación material hasta el proceso psicológico. La imagen se puede formular como condicionante para el comportamiento humano, es ahí donde se constituye como elemento diferenciador dentro de la comunicación política.

La percepción de la imagen se constituye como el primer mensaje que emiten los políticos a sus electores, el mismo contribuye al posicionamiento y aceptación de los líderes y sus partidos en la mente de sus electores, es fundamental construir una imagen basada en principios y valores que exponga al personaje como un representante íntegro, leal y honesto identificado directamente con su público. Un político debe considerar aspectos fundamentales que faciliten articular su imagen y generar influencia electoral, la imagen es la conjugación de todos los aspectos que desean transmitir con el objetivo de persuadir a sus votantes, hay que considerar que el discurso político deberá contener modismos propios con un lenguaje cultural, es decir; la forma en la que se deberá expresar para afianzar la imagen y promover la simpatía con su pueblo.

Según (Álvarez del Blanco, 2011) los mercadólogos deben priorizar la importancia de los sentidos en la percepción de las marcas, la vista es el sentido de mayor percepción en los individuos ocupa el $58 \%$, el olfato $45 \%$, el oído $41 \%$, el gusto $31 \%$ y el tacto $25 \%$ (Gómez \& Mejía, 2012), con estas perspectivas se destaca la relevancia que tiene la construcción estratégica de la imagen dentro del escenario político la cual deberá ser coherente y funcional que logre persuadir a su electorado.

\section{La comunicación de la imagen política}

La construcción de una buena imagen política facilita la comunicación en los medios y ayuda a personalizar su discurso de acuerdo a sus destinatarios, hay que reforzar la imagen de un político para incrementar su notoriedad en las campañas. El despliegue publicitario deberá mostrar al político como una persona ética y de pueblo y que se identifique fácilmente con sus electores (Piana \& Baeza, 2013). Las estrategias y tácticas deberán estar enfocadas en mostrar a una persona y su programa político articulado en relación a su mercado destinado a satisfacer necesidades de su público.

La propaganda política se constituye como la búsqueda permanente de votos, tiempo atrás las formas tradicionales de comunicación de los políticos se centraba en los discursos políticos populares y las visitas puerta a puerta; en la actualidad la gran mayoría utiliza plataformas digitales para acercarse a su electorado tales como: portales en la web, correos electrónicos, ciber navegadores y celulares, facilitando la comunicación y persuasión, las nuevas formas de comunicación y el acceso al internet han beneficiado el desarrollo de las campañas electorales, las plataformas virtuales facilitan la interacción del político con su elector para transmitir ideas, discursos y 


\section{Jornada de Investigación Universidad Tecnológica Empresarial de Guayaquil}

proyectos. La constante evolución de la tecnología obliga a innovar las formas de hacer política para no quedarse rezagado (Castillo, 2016).).

El desarrollo tecnológico facilita la promoción y difusión de la imagen del candidato, la cual le permite crear presencia en la web y generar tráfico en las redes digitales, el uso de estas herramientas ayuda a los políticos a construir su marca personal (Lucas, 2012). El uso de medios de comunicación permite acercar el candidato a sus votantes, las presentaciones televisivas son de suma importancia durante las campañas electorales, permiten evidenciar el esfuerzo que realizan los políticos para generar presencia; la radio centra la atención en el discurso y no en la imagen; la publicidad en la vía pública se fundamenta en el Marketing Político ético, el cual se encarga de promover la imagen positiva de un candidato. La combinación de imagen y sonidos permite la optimización de los recursos informativos para persuadir al electorado (Merino, 2015).

Toda la información que los votantes reciben sobre los candidatos, ayuda a la formación de la imagen y contribuye a la decisión final en las elecciones. El objetivo de la publicidad política es presentar al candidato como modelo del líder perfecto, resaltando sus acciones, discurso y cualidades. Según (Berrocal, 2004, p.58) Por acciones se entiende a los "cambios en la forma de vestir, de hablar, de peinarse 0 retoques estéticos más o menos oficiosos", ya que es importante que "los políticos aprendan a adaptarse a todas las exigencias de la producción y el guion" (Carceller Cobos, 2013).

\section{Método}

Para determinar los factores que influyen la percepción de la imagen de un político en el escenario electoral, se procedió a realizar una investigación de carácter cuantitativa a los ciudadanos del cantón Machala.

Para su ejecución se realizó una proyección de los habitantes del cantón tomando como referencia el censo poblacional del año 2010 realizado por el Instituto Nacional de Estadísticas y Censos (INEC) dando como resultado una población actual de 275378 habitantes; la cual se obtuvo una muestra de 399 personas con un nivel de confiabilidad del $95 \%$ y un margen de error admisible del 5\%. Se elaboró un instrumento de investigación con preguntas cerradas y de opción múltiple, las cuales fueron sometidas a un proceso de validación mediante una prueba piloto; una vez validado el instrumento se realizó el levantamiento de información a los habitantes del cantón Machala, luego se procedió a realizar la tabulación de datos obtenidos para su posterior presentación en gráficos estadísticos.

\section{Resultados}

De acuerdo a los datos obtenidos en la presente investigación que tiene por objetivo determinar los factores que influyen en la percepción de la imagen de un político, se puede identificar que el $89 \%$ de la población del cantón Machala si se interesa por temas relacionados a la política en el país, mientras que existe un $11 \%$ de personas se muestran renuentes a tratar estos temas. El $80 \%$ declaró no pertenecer a ningún partido político por otro lado el $20 \%$ de la población afirma que si mantiene cierta preferencia. El $17 \%$ posee cierta afinidad por partidos de derecha y un $7 \%$ se inclina hacia partidos de izquierda, las personas que se mantiene en el centro representan un $4 \%$ de la muestra investigada; finalmente un $72 \%$ no destacan ninguna tendencia política de preferencia. Cabe destacar que para disipar dudas se realizó una breve 145 


\section{Jornada de Investigación Universidad Tecnológica Empresarial de Guayaquil}

explicación sobre los partidos políticos y las tendencias políticas existentes en el país.

Por otro lado, el $87 \%$ de los encuestados considera importante informarse sobre las actividades realizadas por los políticos; a su vez un 38\% las personas prefieren la televisión como un medio masivo para informarse, seguido del $23 \%$ que prefiere la radio, rezagando a la prensa con un $19 \%$. Se consultó a los ciudadanos si utilizan las redes sociales para seguir a detalle a sus políticos favoritos dando como resultado que el $68 \%$ de las personas siguen a los políticos de su preferencia en medios sociales mientras que el $32 \%$ son personas que no tienen cuentas sociales (off line). De las personas que siguen a los políticos en la web el $56 \%$ resaltan que Facebook es la red social de mayor preferencia seguido por Twitter con un $16 \%$ de aceptación.

El $88 \%$ de las personas consideran muy importante que los políticos deben fortalecer su imagen para fidelizar a su electorado, mientras que el $69 \%$ de las personas afirman que la imagen que promueve un político motiva su interés.

Las expectativas que se espera de los políticos actuales el $24 \%$ de la muestra manifiesta que la formación académica es de vital importancia seguido del $19 \%$ que se inclina por la experiencia demostrada durante el ejercicio de la función pública, un $18 \%$ espera que un político demuestre credibilidad en sus actos y en su discurso político; estos datos coinciden al contrastar con los factores que motivan su preferencia hacia un determinado político, dando como resultado que el $36 \%$ de los encuestados destaca la experiencia que promueve un político seguido del $26 \%$ a la vigencia política que ha mantenido en el tiempo y el $14 \%$ considera que la credibilidad es una de las razones fundamentales para la predilección de un político.

El $47 \%$ de las personas proponen que el factor que influye en la percepción de la imagen es que el político domine su lenguaje oral, visual y simbólico seguido del $18 \%$ que consideran que la vigencia política repercute de forma positiva en la percepción de la imagen; mientras que el $15 \%$ expresa que los cargos que ha desempeñado fortalecen la imagen del político. Los sentidos se involucran en la percepción de la imagen política, de acuerdo a la investigación realizada el $85 \%$ de las personas afirman que la vista es el sentido más importante para influir políticamente al electorado. A su vez el $46 \%$ de las personas manifestaron que los factores que influyen de forma negativa en la percepción de la imagen es el enriquecimiento ilícito, el $37 \%$ que supone afecta directamente al político cuando este se cambia constantemente de partido político, un $12 \%$ opina que los escándalos amorosos en los que se han visto involucrados los políticos afectan en gran manera su imagen pública.

\section{Discusión}

Existe una estrecha relación entre las ciencias políticas y el marketing; siendo esta última el instrumento que ayuda a los políticos a promover sus propuestas, su gobierno y su imagen al público objetivo. Las herramientas de marketing han sido un apoyo indispensable para dirigir campañas de promoción de candidatos y sus partidos políticos. Los políticos que aplican estas herramientas poseen cierta ventaja competitiva pudiendo diferenciarse de sus equivalentes en el escenario electoral.

Los habitantes del cantón Machala manifestaron no tener una tendencia política de preferencia según el $72 \%$ de la muestra investigada, en este mismo sentido el $28 \%$ 


\section{Jornada de Investigación Universidad Tecnológica Empresarial de Guayaquil}

dice si sentirse identificado por una tendencia política; de los cuales un 17\% mantiene preferencia por partidos de derecha, un $7 \%$ por partidos de izquierda y posteriormente el $4 \%$ de las personas conservan su posición central. Un gran número de personas no pertenecen a ningún partido político y no tiene definida una tendencia política, evidenciando el escaso trabajo de los partidos políticos para atraer simpatizantes.

La imagen es un tema que ha promovido la discusión entre los diferentes grupos, estableciendo que un político debe tener una identidad que lo caracterice para destacar sus cualidades; pudiendo confirmar que la imagen que promueve es el primer mensaje que emite a su público, por tal razón las personas consideran de gran importancia construir una buena imagen política que ayude promover el interés y fidelizar a su electorado.

Los encuestados considera importante informarse sobre las actividades que realizan los políticos, pese al crecimiento de la tecnología las personas siguen optando por la televisión como medio preferido de información. El internet se ha convertido es un medio eficaz para propagar temas políticos en la web siendo facebook la red social con mayor uso por parte de las personas para seguir a los políticos.

Las expectativas que esperan los electores es la formación académica que posea los políticos y la experiencia demostrada durante el ejercicio de la función pública, a su vez la credibilidad demostrada en sus actos y en su discurso político ayudan a afianzar su imagen política. El factor que influye en la percepción de la imagen es que un político domine su lenguaje oral, visual y simbólico para atraer simpatizantes, la vigencia política fortalece la construcción de la imagen pública, los cargos que ha desempeñado contribuyen a su aceptación en la palestra electoral.

El éxito de un político en el escenario electoral se debe al interés de las personas en temas relacionados a la política, cuando un mercado posee una opinión fragmentada difícilmente acepta las propuestas emitidas, en todo caso es importante desarrollar estrategias que permitan acercar al político con sus votantes. Los sentidos se involucran en la percepción de la imagen política un gran número de personas consideran que la vista es el sentido más importante para influir políticamente al electorado.

Los factores que afectan de forma negativa en la percepción de la imagen es el enriquecimiento ilícito lo cual ha denigrado por completo la imagen pública de determinados personajes, cuando un político se cambia constantemente de partido comúnmente conocido como el "camisetazo" perjudica la imagen y aleja a los votantes, un político se vuelve un personaje mediático y su honorabilidad se ve afectada cuando este se ha visto involucrado en escándalos amorosos.

Los factores principales que motivan la preferencia hacia un determinado político es la experiencia demostrada en el ejercicio de sus funciones, la vigencia política es de suma importancia logrando mantener la imagen y el nombre del candidato en la mente de sus electores, la credibilidad es una de las razones fundamentales para la predilección de un político. 
Revista Ciencia \& Tecnología No. 16, 31 de octubre de 2017 ISSN impreso: 1390 - 6321

\section{Jornada de Investigación Universidad Tecnológica Empresarial de Guayaquil}

\section{Referencias bibliográficas}

Alonso Coto, M., \& Adell, Á. (2011). Marketing político 2.0. Grupo Planeta.

Álvarez del Blanco, R. (2011). Branding hoy: estrategias que funcionan. Marca multisensorial, espléndidamente lúcida. p26 a 33. Harvard Deusto. Marketing \& Ventas, No 106.

Barranco, J. (2012). Marketing Político y Electoral. Madríd: Pirámide.

Barrientos, P. (2010). El marketing del partido político en el gobierno. Recuperado de https://www.redalyc.org/pdf/1650/165014341006.pdf

Benítez M. A., \& Salas L, E. (2016). Estudio de estrategias de marketing político en el partido político "Alianza País" de Ecuador. Revista Contribuciones a las Ciencias Sociales (Eumed).

Bustamante, C. A., Blanc, G. R., \& Espinoza, L. E. (2015). El estado político actual del Ecuador después de múltiples procesos electorales: los factores de decisión, la influencia del electorado y el impacto del marketing político. Revista EconPapers. Recuperado https://econpapers.repec.org/article/ervcoccss/y_3a2015_3ai_3a201502_3a16.htm

Carceller Cobos, J. (2013). La videopolítica en campaña evolución del spot electoral en España entre 2004 Y 2011. Vivat Academia, 124:1-20.

Capriotti, P. (2013). Planificación estratégica de la imagen corporativa. Instituto de Investigación en Relaciones Públicas.

Castillo G. D. (2016). La campaña electoral del candidato Nicolás Maduro en la cobertura mediática del diario impreso Correo del Orinoco. Eumed.

Castro M. L. (2012). El marketing político en Estados Unidos: el caso Obama. Recuperado http://www.scielo.org.mx/scielo.php?script=sci_arttext\&pid=S187035502012000100008.

Costa, J. (2003), Creación de la imagen corporativa. El paradigma del siglo XXI, en Razón y Palabra [en línea], núm. 34, disponible en: http://www.razonypalabra.org.mx/anteriores/n34/jcosta.html, recuperado: 28 de mayo de 2007

García, M. A., \& Jaimes, L. L. (2010). Influencia del marketing electoral en la alternancia política del municipio de Toluca. Espacios Públicos, 13 (29): 48-66.

Gómez, R., C., \& Mejía, J. (2012). La gestión del Marketing que conecta con los sentidos. Recuperado de https://www.redalyc.org/pdf/206/20625032010.pdf

Kotler, P., \& Armstrong, G. (2012). Fundamentos de Marketing. México: Pearson 
Revista Ciencia \& Tecnología No. 16, 31 de octubre de 2017 ISSN impreso: 1390 - 6321

\section{Jornada de Investigación Universidad Tecnológica Empresarial de Guayaquil}

Educación.

Lerma, A. E., Bárcena, S., \& Vite, R. (2011). Marketing Politico. Cengage Learning.

Lucas, M. A. (2012). Campaña en la Red. Estrategias de Marketing. REDMARKA UIMA, 195.

Mc Phail Fanger, E. (2014). Análisis de la imagen en campañas políticas. Recuperado de https://www.redalyc.org/pdf/1995/199530728047.pdf

Merino, L. J. (2015). El arte y la ciencia de la persuasión ¿es indispensable el marketing político en américa latina? Recuperado de https://econpapers.repec.org/article/ervobserv/y_3a2015_3ai_3a209_3a02.htm.

Orejuela, S. (2009). Personalización Política, la Imagen del político como estrategia electoral. Dialnet, Revista de Comunicación.

Piana, R., \& Baeza, N. (2013). Candidatos a medida ¿Cómo se construyó el candidato que le ganó a los Kirchner? Recuperado de https://www.redalyc.org/pdf/1514/151430876009.pdf

Ponce, M., Besanilla, T., \& Rodríguez, H. (2012). Factores que influyen en el comportamiento del consumidor. Madrid: Eumed.

Real Academia Española. (2014). Diccionario Real Academia Española. Recuperado el 15 de noviembre de 2016, de http://dle.rae.es/?id=KzwDY4y

Richard, E. (2008). Álvaro Uribe: la comunicación por la imagen. Principios de marketing político. Dialnet, Revista Opera.

Salas, L. E., \& Serratore, N. (2015). Análisis de herramientas de marketing político utilizadas en campañas presidenciales del Ecuador (período 1984 - 2008). Madrid: Eumed.

Sánchez, J. D. (2013). Votantes que consumen, consumidores que votan. Más poder local.

Sanmartin, A. (2007). El Poder del cuarto poder. La influencia del marketing político en las democracias modernas. Dialnet, Revista Estudios centroamericanos.

Stanton, W., Etzel, M., \& Walker, B. (2007). Fundamentos de Marketing. México: McGraw-Hill Interamericana Editores S.A.

Villafañe, J. (2002). Imagen positiva. Gestión estratégica de la imagen de las empresas. Madrid: Pirámide. 